\title{
ASSOCIAÇÃO ENTRE INSEGURANÇA ALIMENTAR E CONSUMO ALIMENTAR DE IDOSOS ASSISTIDOS POR UMA ESTRATÉGIA DE SAÚDE DA FAMÍLIA DO INTERIOR PAULISTA
}

\author{
Juliana Santos Bóia, Vitoria Eduarda Fernandes de Morais, Aparecido Ignacio Junior, Sabrina Alves \\ Lenquiste, Rayana Loch Gomes
}

Universidade do Oeste Paulista - UNOESTE, Presidente Prudente, SP. E-mail: julianinha.santos15@gmail.com

\section{RESUMO}

O objetivo foi verificar a associação entre insegurança alimentar (IA) e consumo alimentar de idosos assistidos por uma Estratégia da Saúde da Família (ESF). Foram avaliados 16 idosos, de ambos os sexos, com média de idade 69,13 anos e IMC de $29,23 \mathrm{~kg} / \mathrm{m}^{2}$. Foram utilizados a Escala brasileira de insegurança alimentar, questionário de frequência alimentar e recordatório alimentar de 24 horas. Peso e altura foram retirados dos prontuários dos pacientes. Observou-se que 37,5\% dos idosos estavam em IA leve, 31,25\% em IA moderada, $18,75 \%$ em IA grave e apenas 12,5\% em segurança alimentar. Os indivíduos não consomem leite desnatado e possuem frequente ingestão de ovos, embutidos, margarina, cereais refinados, bebidas industrializadas, doces e balas. E pouca ou nenhuma utilização de azeite e cereais integrais. Sem associação significante entre IA e consumo alimentar. Conclui-se que não houve associação entre insegurança alimentar e consumo alimentar de idosos assistidos por uma ESF.

Palavras-chave: alimentos, envelhecimento, segurança alimentar, assistência alimenta, saúde pública.

\section{ASSOCIATION BETWEEN FOOD INSECURITY AND FOOD CONSUMPTION OF ELDERLY PEOPLE ASSISTED BY A FAMILY HEALTH STRATEGY IN THE INTERIOR OF SÃO PAULO.}

\begin{abstract}
The aim was to verify the association between food insecurity (FI) and food consumption of elderly people assisted by a Family Health Strategy (FHE). Sixteen elderly people, of both sexes, with a mean age of 69.13 years and BMI of $29.23 \mathrm{~kg} / \mathrm{m}^{2}$ were evaluated. The Brazilian Food Insecurity Scale, food frequency questionnaire and 24-hour food recall were used. Weight and height were taken from the patients' records. It was observed that $37.5 \%$ of the elderly were in mild $\mathrm{Al}, 31.25 \%$ in moderate $\mathrm{Al}, 18.75 \%$ in severe $\mathrm{Al}$ and only $12.5 \%$ in food security. Individuals do not consume skimmed milk and have frequent intake of eggs, sausages, margarine, refined cereals, industrialized beverages, sweets and candies. And little or no use of olive oil and whole grains. No significant association between Al and food consumption. It is concluded that there was no association between food insecurity and food consumption in elderly people assisted by an ESF.
\end{abstract}

Keywords: food, aging, food safety, food assistance, public health.

\section{INTRODUÇÃO}

Atualmente, não somente em países desenvolvidos, mas também em subdesenvolvidos, é perceptível a constante transição demográfica, na qual tem resultado no declínio percentual da taxa de fertilidade, e no aumento significativo da taxa de envelhecimento populacional $^{1}$.
O envelhecimento é o processo irreversível de diminuição orgânica e funcional da vida, resultado da progressão dos anos. Para esse processo, deve-se levar em consideração não somente o fator biológico, mas também o social, psicológico e cultural. Acomete todos os seres humanos, sem distinção alguma, e varia de indivíduo para indivíduo, de acordo com 
determinação genética, influência do seu estilo de vida, hábitos alimentares e ambiente em que se vive. É no envelhecimento que ocorrem diminuições dos aspectos funcionais do indivíduo, que levam a uma diminuição da capacidade de adaptação ao meio ambiente e resulta em uma maior predisposição ao desenvolvimento de doenças, como doenças cardiovasculares, Diabetes, Osteoporose, Alzheimer entre outras ${ }^{2,3}$.

As modificações associadas ao envelhecimento, ocorrem em diversos sistemas corporais como o sistema cardíaco, hormonal, respiratório e musculoesquelético. Dentre as principais alterações, encontra-se a mudança na composição corporal que apresenta grande relação com o estado nutricional de idosos. Também pode ocorrer um aumento considerável da massa de gordura e uma diminuição significativa da massa muscular e óssea, afetando em grande escala membros inferiores, o que leva a perdas funcionais, processo esse caracterizado como Sarcopenia, podendo fazer com que o indivíduo apresente restrições sociais e dependências, além de elevar os custos com a saúde ${ }^{3,4}$.

Com o passar da idade, o metabolismo se torna mais vulnerável, decorrente a fatores que influenciam na qualidade de vida, contribuindo para uma menor absorção dos nutrientes. Percepções sensoriais como visão, paladar e olfato entram em declínio também e por conta disso, o padrão alimentar do idoso muitas vezes é inadequado ${ }^{5}$.

Os aspectos dietéticos e nutritivos têm papel fundamental no que diz respeito a promoção, manutenção, recuperação da saúde, estado nutricional do idoso e da população em geral. Com esta finalidade, os fatores que afetam o hábito alimentar podem ser controlados, elevando assim, a qualidade de vida da terceira idade. Um dos fatores fisiológicos que acometem a ingestão alimentar é a falta de apetite, contribuindo para menor absorção de vitaminas, minerais e outros nutrientes, levando a depleção nutricional $^{6,7}$.

Outro fator importante é a insegurança alimentar (IA) , que é caracterizada pela ausência da capacidade de adquirir os alimentos nutricionais seguros e adequados, de forma sociável. Há uma violação nos direitos humanos quando o cidadão não recebe a alimentação em quantidade e qualidade ideal. Como consequência dessa insegurança, há um aumento do aparecimento de doenças advindas da má nutrição, causando assim uma queda na qualidade de vida. Ou seja, a insegurança alimentar pode levar a uma alimentação inadequada prejudicando o estado nutricional, levando a desnutrição ou a obesidade, atingindo diretamente a saúde da população ${ }^{8,9}$.

Segundo a literatura o fator que mais se associa a IA é a questão financeira, devido a renda familiar muitos idosos deixam de ter acesso aos alimentos em qualidade e quantidade suficiente $^{10}$.

Ainda, outro fator de grande relevância para o surgimento da IA no idoso é a vulnerabilidade social apresentada por esse público. Essa vulnerabilidade é caracterizada pela falta de informações confiáveis, acesso aos meios de comunicação, escolaridade, influência sobre as decisões políticas, cultura, segurança e bem-estar. No Brasil, idosos vivem com recursos limitados para manter toda uma família, sendo muita das vezes uma aposentadoria de baixo valor, promovendo uma condição social inadequada ${ }^{11,12}$.

Outro fator que tem contribuído para o desenvolvimento da insegurança alimentar não só na população idosa, mas também na população em geral é a pandemia desencadeada pela COVID-19 a qual gerou desempregos, aumento no preço do aluguel, água, energia elétrica, bem como no preço dos alimentos, o que gerou um grande impacto na renda familiar fazendo com que muitas famílias reduzissem a quantidade ingerida de alimentos, prejudicando consequentemente a qualidade nutricional das refeições ${ }^{13}$.

A avaliação precoce dos idosos é uma ação necessária para a verificação da IA permitindo assim intervenções a modo de evitar consequências futuras nessa população causadas por essa insegurança. Para isso pode ser utilizada a Escala Brasileira Insegurança Alimentar (EBIA), a qual foi criada por um grupo de pesquisadores com o objetivo de produzir uma ferramenta capaz de medir diretamente a segurança alimentar e nutricional no Brasil. A EBIA contém 14 questões estabelecendo uma pontuação a modo de classificar o nível de $I A^{14}$.

A IA é um dos principais fatores que altera o consumo alimentar de idosos, que como consequência disso aumenta os números de casos de doenças nessa população, tais com a obesidade e desnutrição grave ${ }^{9,10}$. Portanto, o objetivo do presente estudo foi verificar a 
associação entre IA e consumo alimentar de idosos assistidos por uma Estratégia da Saúde da Família (ESF) em uma cidade do interior paulista.

\section{MÉTODOS}

Foi realizado um estudo descritivo de caráter transversal, desenvolvido com os idosos assistidos em uma ESF de Presidente PrudenteSP. O projeto foi devidamente cadastrado e aprovado na Plataforma Brasil (CAEE: 34316720.0.0000.5515) e no Comitê de Ética em Pesquisa (6294) da UNOESTE.

\section{Participantes}

Para conduzir este estudo, foram recrutados indivíduos que possuem idade igual ou maior que 60 anos, de ambos os sexos, que residem no bairro assistido pela ESF. A avaliação foi realizada no domicílio do participante, onde as pesquisadoras ficaram do lado de fora da casa do mesmo mantendo distância segura entre as pesquisadoras e os avaliados devido a COVID-19. Para compor o grupo os integrantes deviam atender aos seguintes critérios de inclusão: 1 ) Não estar acamado, 2) Possuir capacidade cognitiva para compreender e realizar os procedimentos do estudo, 3) $\mathrm{Em}$ caso de analfabetismo estar acompanhado de alguém de sua confiança.

Os indivíduos incapazes de compreender os procedimentos do presente estudo e os que não aceitaram responder os questionários não foram incluídos na amostra. Este estudo avaliou 16 voluntários.

\section{Procedimento experimental}

Inicialmente as pesquisadoras fizeram um levantamento dos indivíduos assistidos na ESF que possuíam idade igual ou acima de 60 anos. Em seguida, coletaram os dados descritos no prontuário, como comorbidades, medicamentos ingeridos, peso, altura e endereço residencial. Após essa etapa, as pesquisadoras foram até a residência dos mesmos, porém ficaram do lado de fora, mantendo uma distância segura e usaram a máscara modelo N95/PFF2 recomendada pelo Ministério da Saúde, em seguida se apresentaram como acadêmicas do curso de nutrição da Unoeste, explicaram que sobre a pesquisa e esses idosos foram convidados a participarem do estudo.

As pesquisadoras leram detalhadamente - TCLE para os idosos e os que aceitaram participar da pesquisa assinaram o TCLE. Para assinar o TCLE, foi entregue uma caneta para cada idoso, a qual ficou com o mesmo, sendo higienizada na hora da entrega. Em seguida, as pesquisadoras levantaram questionamentos de alguns dados, aplicaram a Escala Brasileira de Insegurança Alimentar (EBIA), o Recordatório alimentar de 24 horas (R24h) e o Questionário de frequência alimentar (QFA).

\section{Escala Brasileira de Insegurança Alimentar (EBIA)}

A EBIA é uma ferramenta utilizada para a identificação da segurança e insegurança alimentar e nutricional na população Brasileira, sendo composta por 14 questões diretas referente ao consumo alimentar e renda no domicílio nos últimos três meses. É atribuída uma pontuação para cada questão respondida obtendo-se uma classificação que é caracterizada em dois grupos, sendo eles, domicílios que apresentam menores de 18 anos e domicílios sem menores de 18 anos. Em ambos apresentam quatro subdivisões, que são elas, segurança alimentar (SA), insegurança alimentar leve (IL), insegurança alimentar moderada (IM) e insegurança alimentar grave (IG). Nos domicílios com menores de 18 anos, a pontuação será classificada da seguinte maneira: 0 pontos (SA); 1-5 pontos (IL); 6-9 pontos (IM) e 10-14 pontos (IG). No caso de domicílios sem menores de 18 anos, a pontuação será: 0 pontos (SA); 1-3 pontos (IL); 4-5 pontos (IM) e 6-8 pontos (IG) ${ }^{14}$.

\section{Recordatório alimentar de $\mathbf{2 4}$ horas (R24h)}

O R24h consiste em definir e quantificar todos os alimentos e bebidas ingeridas no período anterior à entrevista, que podem ser as 24 horas precedentes ou, mais comumente, o dia anterior. Esse método foi escolhido por ser de rápida aplicação e imediata recordação dos participantes, condições que predispõem a uma maior participação do indivíduo. Esse método verifica a dieta atual, estimando valores absolutos ou relativos da ingestão de energia e nutrientes amplamente distribuídos no total de alimentos oferecidos ao indivíduo ${ }^{15}$.

O R24h foi aplicado nos dias de terça ou sexta-feira da seguinte maneira: as pesquisadoras questionaram os participantes da pesquisa a respeito das refeições realizadas no dia anterior e dos alimentos contidos em cada uma delas e anotaram. Devido a pandemia foi possível realizar apenas uma coleta. Com as informações obtidas, foi realizado o cálculo das 
calorias ingeridas, bem como porcentagem de macronutrientes por meio do programa Dietbox.

\section{Questionário de Frequência Alimentar (QFA)}

O questionário de frequência de consumo alimentar foi desenvolvido a partir do questionário validado por Sichieri \& Everhar $^{16}$. É um dos métodos mais utilizados para mensurar o consumo alimentar em estudos. Este instrumento apresenta uma tabela de alimentos mais consumidos frequentemente ou um padrão alimentar de uma determinada população, registrando assim, a frequência habitual da sua alimentação em um determinado período ${ }^{17}$.

\section{Tratamento e análise dos resultados}

A caracterização da amostra e de alguns dados referentes ao consumo alimentar foi realizada por meio de estatística descritiva e os resultados foram apresentados em valores de média, desvio padrão, mínimo, máximo e porcentagens. Para associação entre grau de insegurança alimentar e consumo alimentar dos indivíduos pelo recordatório alimentar, primeiramente foi verificada normalidade dos dados por meio do teste de Shapiro-wilk seguido de teste de correlação de Pearson para dados paramétricos e de Spearman para dados não paramétricos. Todas as análises estatísticas foram realizadas utilizando o software Statistical Package for the Social Sciences (SPSS) versão 16.0 e a significância estatística adotada foi de $5 \%$.

\section{RESULTADOS}

A caracterização da amostra total ( $n$ = 16), por meio de valores médios com seus respectivos desvios padrões e/ou porcentagens para idade, variáveis antropométricas, gênero, membros da família, número de refeições realizadas por dia, comorbidades associadas e utilização de medicamentos pode ser observada na tabela 1.

Tabela 1. Caracterização da amostra total $(n=16)$.

\begin{tabular}{|c|c|}
\hline Variáveis & \\
\hline Idade (anos) média \pm DP (min-max) & $69,13 \pm 5,26(62-79)$ \\
\hline Peso (Kg) média \pm DP (min-max) & $71,29 \pm 21,11(46,6-133,7)$ \\
\hline Altura (m) média \pm DP (min-max) & $1,57 \pm 0,09(1,43-1,73)$ \\
\hline ÍMC $\left(\mathrm{Kg} / \mathrm{m}^{2}\right)$ média \pm DP (min-max) & $29,23 \pm 10,29(17,76-61,04)$ \\
\hline \multicolumn{2}{|l|}{ Gênero } \\
\hline Feminino, $n(\%)$ & $12(75)$ \\
\hline Masculino, $n(\%)$ & $4(25)$ \\
\hline \multicolumn{2}{|l|}{ Membros na família } \\
\hline $1, n(\%)$ & $6(37,5)$ \\
\hline $2, n(\%)$ & $9(56,25)$ \\
\hline $5, n(\%)$ & $1(6,25)$ \\
\hline \multicolumn{2}{|l|}{ Número de refeições no dia } \\
\hline $1-2, n(\%)$ & $3(18,75)$ \\
\hline $3-4, n(\%)$ & $9(56,25)$ \\
\hline $5-6, n(\%)$ & $4(25)$ \\
\hline \multicolumn{2}{|l|}{ Presença de comorbidades } \\
\hline Hipertensão arterial, $n$ & $11(68,75)$ \\
\hline Diabetes mellitus, $n$ & $5(31,25)$ \\
\hline Dislipidemias, $n$ & $4(25)$ \\
\hline outras, $n$ & $14(87,5)$ \\
\hline
\end{tabular}

Fonte: Os autores.

A figura 1 reporta a classificação da escala EBIA. Nos domicílios sem moradores menores de 18 anos 15,38\% indivíduos apresentaram SA, 38,46\% IL, 30,77\% IM e 15,38 IG, enquanto nos domicílios com moradores menores de 18 anos, em um total de 3 domicílios, houve a presença de $33 \%$ de IL, e o mesmo para
IM e IG. No total, houve $12,5 \%$ em SA, 37,5\% em IL, 31,25\% em IM e $18,75 \%$ em IG. 


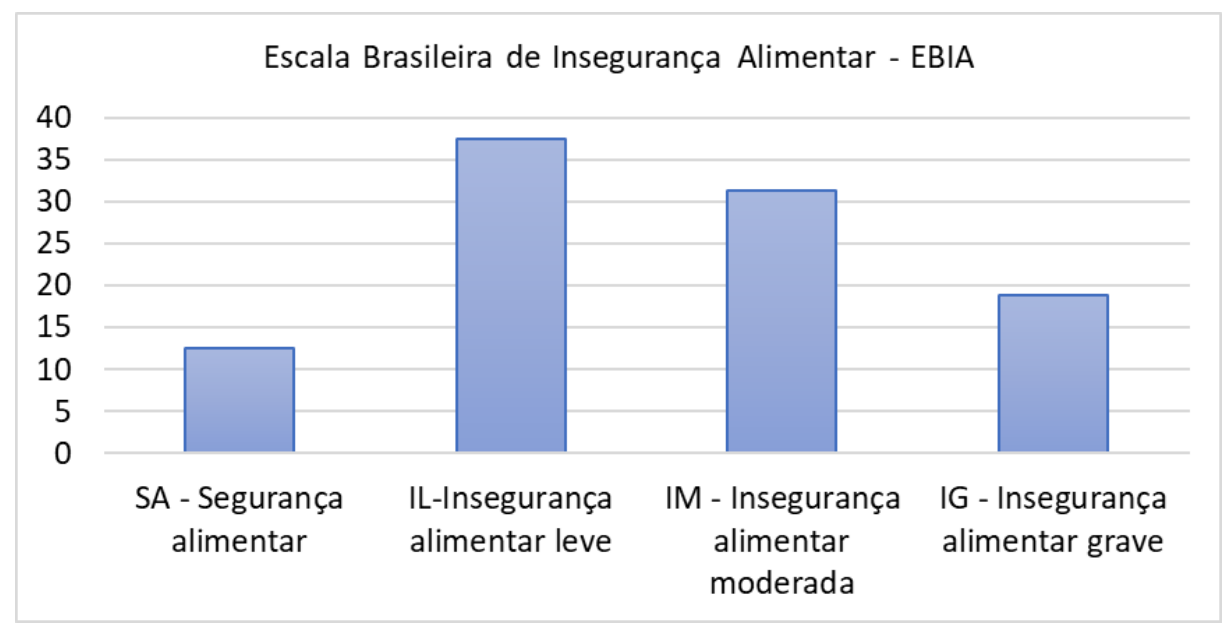

Figura 1. Classificação da EBIA na população do estudo. Fonte: Os autores.

A tabela 2 apresenta a associação entre valores de EBIA e consumo alimentar em calorias, porcentagem de carboidratos, proteína e lipídeos. Observa-se que não houve associação estatisticamente significativa entre os parâmetros avaliados.

Tabela 2. Correlação entre valores da Escala brasileira de insegurança alimentar (EBIA) e consumo alimentar $(n=16)$.

\begin{tabular}{lcc}
\hline \multirow{2}{*}{ Variáveis independentes } & \multicolumn{2}{c}{ Correlação (variável dependente: EBIA) } \\
\cline { 2 - 3 } & $\mathbf{R}$ & $\boldsymbol{p}$-valor \\
\hline Calorias/dia & $-0,094$ & $0,729^{*}$ \\
Proteína (\%) & 0,255 & $0,341^{* *}$ \\
Carboidrato (\%) & 0,015 & $0,957^{* *}$ \\
Lipídio (\%) & $-0,291$ & $0,275^{*}$ \\
\hline
\end{tabular}

\section{Fonte: Os autores.}

Legenda: *Correlação de Pearson; **Correlação de Spearman

$\mathrm{Na}$ tabela 3 observam-se os valores médios e seus respectivos desvios padrão do consumo alimentar em calorias e porcentagem de macronutrientes, carboidratos, proteínas e lipídeos dos indivíduos divididos em grupos pela classificação da EBIA. 
Tabela 3. Consumo alimentar em calorias e porcentagem de macronutrientes entre os grupos classificados pela EBIA $(n=16)$.

$\begin{array}{lcccc} & \begin{array}{c}\text { Segurança } \\ \text { alimentar }\end{array} & \begin{array}{c}\text { Insegurança } \\ \text { alimentar leve }\end{array} & \begin{array}{c}\text { Insegurança } \\ \text { alimentar } \\ \text { moderada }\end{array} & \begin{array}{c}\text { Insegurança } \\ \text { alimentar grave }\end{array} \\ \text { Calorias } & 1036,79 \pm & 809 \pm & 1080,20 \pm & 697,00 \pm \\ \text { Carboidrato } & 181,39 & 163,01 & 267,50 & 200,19 \\ \text { Proteína } & 59,41 \pm & 45,88 \pm & 49,99 \pm & 60,80 \pm \\ \text { Lipídeos } & 3,44 & 12,61 & 14,63 & 6,70 \\ & 11,56 \pm & 20,31 \pm & 19,08 \pm & 16,93 \pm \\ \text { Fonte: Os autores } & 1,52 & 6,64 & 7,18 & 3,78 \\ & 29,04 \pm & 33,81 \pm & 30,91 \pm & 22,28 \pm \\ & 1,92 & 6,38 & 7,82 & 5,74\end{array}$

A tabela 4 demostra a frequência do consumo alimentar de grupos de alimentos considerados promotores e protetores da obesidade, sendo a frequência estabelecida por meio de porcentagem.

Tabela 4. Frequência de consumo alimentar dos participantes $(n=16)$.

\begin{tabular}{|c|c|c|c|c|c|}
\hline Alimentos & Diário & $\begin{array}{c}1 \mathrm{x} / \\
\text { semana }\end{array}$ & $\begin{array}{c}2-4 x / \\
\text { semana }\end{array}$ & $\begin{array}{l}1-3 x / \\
\text { mês }\end{array}$ & $\begin{array}{c}\text { (continua) } \\
\text { Não } \\
\text { consome }\end{array}$ \\
\hline & $\%$ & $\%$ & $\%$ & $\%$ & $\%$ \\
\hline \multicolumn{6}{|l|}{ Leite e derivados } \\
\hline $\begin{array}{l}\text { Leite desnatado ou semi- } \\
\text { desnatado }\end{array}$ & - & - & - & - & 100 \\
\hline Leite integral & 56,25 & 6,25 & 31,25 & 6,25 & - \\
\hline logurte & - & - & - & - & 100 \\
\hline $\begin{array}{l}\text { Queijo branco } \\
\text { (minas/frescal) }\end{array}$ & - & 25 & - & 25 & 50 \\
\hline $\begin{array}{l}\text { Queijo amarelo } \\
\text { (prato/mussarela) }\end{array}$ & - & 50 & 25 & 18,75 & 6,25 \\
\hline \multicolumn{6}{|l|}{ Carnes e ovos } \\
\hline Ovo frito & 18,75 & 6,25 & 75 & - & - \\
\hline Ovo cozido & 18,75 & 50 & 18,75 & 12,5 & - \\
\hline Carne de boi & - & 68,75 & - & 25 & 6,25 \\
\hline Carne de porco & - & - & - & 25 & 75 \\
\hline Frango & - & 75 & 25 & - & - \\
\hline Peixe Fresco & - & - & - & - & 100 \\
\hline Embutidos & 6,25 & 31,25 & 62,5 & - & - \\
\hline \multicolumn{6}{|l|}{ Óleos } \\
\hline Azeite & - & - & - & - & 100 \\
\hline Manteiga & - & - & - & - & 100 \\
\hline Margarina & 68,75 & 6,25 & 25 & - & - \\
\hline Maionese & - & 12,5 & - & 68,75 & 18,75 \\
\hline
\end{tabular}

Petiscos e enlatados

Sanduíches, Pizza, Esfiha,

Salgadinhos, Amendoim) 
Tabela 4. Frequência de consumo alimentar dos participantes $(n=16)$.

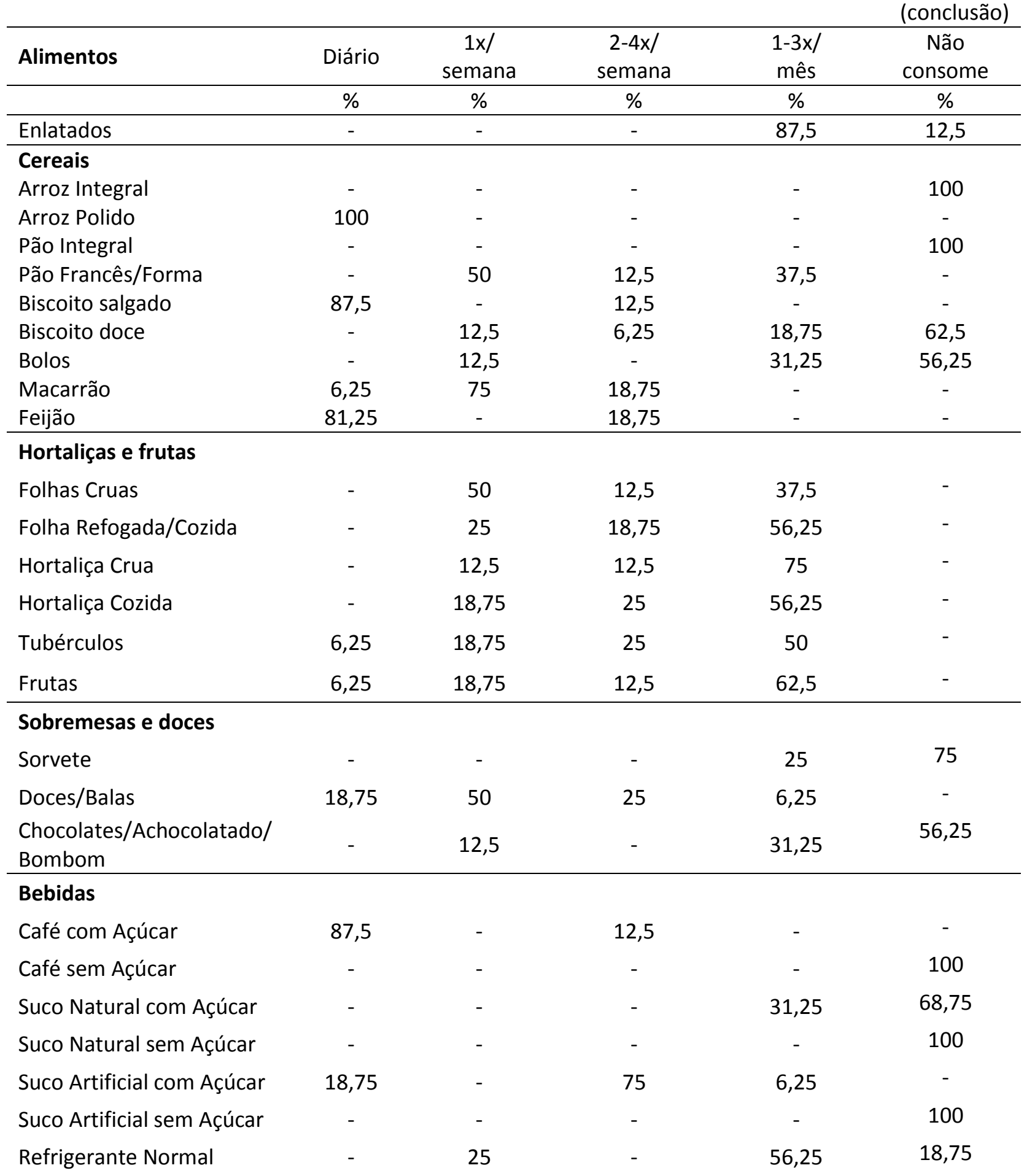

\section{DISCUSSÃO}

O objetivo deste trabalho foi verificar à associação entre IA e o consumo alimentar de idosos assistidos pela por uma Estratégia de Saúde da Família em uma cidade do interior paulista. Nesse estudo a média de idade dos participantes foi acima de 69 anos, mostrando que os indivíduos são idosos jovens.

Em relação a EBIA, essa indicou insegurança alimentar na maioria das residências avaliadas, esse método é o mais utilizado na literatura para avaliar o grau de $I^{14}$. Um estudo que avaliou a IA através da EBIA em 427 idosos com idade igual e superior a 60 anos que residiam em Campinas, mostrou uma maior chance de IA leve entre idosos que tinham renda familiar total igual ou inferior a dois saláriosmínimos. Além disso, observou-se que os idosos com IA possuíam o pior estado nutricional, baixas condições socioeconômicas e maior 
chance do desenvolvimento de doenças crônicas ${ }^{15}$.

A IA possui relação com a baixa renda e - número de moradores presentes na residência, ou seja, quanto maior a quantidade de indivíduos maior será a demanda financeira para suprir as necessidades dos mesmos ${ }^{18}$. No presente estudo os idosos moravam em um bairro de alta vulnerabilidade, porém em sua maioria moravam somente com mais uma pessoa ou até mesmo sozinhos. Alguns idosos podem apresentar dificuldade em se locomover devido as modificações fisiológicas que acontecem no processo de envelhecimento, como por exemplo a redução da capacidade funcional, fazendo com que eles necessitem da ajuda de outras pessoas para a realização das atividades diárias, como ir ao supermercado fazer $\operatorname{compras}^{19}$. Outro fator de grande influência que faz com que os idosos apresentem receio de sair de suas residências é a presença do vírus SARS-CoV-2 mais conhecido como Coronavírus, o qual está se disseminando demasiadamente entre a população de todo o mundo, sendo os idosos indivíduos mais suscetíveis a se contaminarem ${ }^{20}$.

Referente ao consumo alimentar dos indivíduos avaliados, foi verificado no recordatório alimentar baixa ingestão de frutas, verduras, legumes, carnes, maior intervalo entre as refeições e alimentação pouco variada o que também foi encontrado em outro estudo que avaliou a ingestão alimentar. No QFA foi possível verificar a frequência da ingestão de alguns alimentos pertencentes a determinados grupos. dentre esses, destacou-se o grupo do leite e derivados onde os indivíduos não consumiam leite desnatado ou semidesnatado, sendo mais frequente o consumo do leite integral. Ainda, verificou-se uma melhor aceitação do queijo amarelo em relação ao queijo branco. Outro ponto a ser destacado foi a frequente ingestão de ovos, embutidos, margarina, cereais refinados, bebidas industrializadas, doces e balas. Também averiguou-se que nenhum dos participantes da pesquisa utilizavam o azeite e consumiam cereais integrais, como por exemplo o arroz integral ${ }^{21}$.

Em geral acredita-se que os indivíduos que possuem IA vão apresentar baixo peso e desnutrição devido ao pouco acesso aos alimentos, no entanto o estudo de Brito et al. mostrou uma associação entre IA, sobrepeso e obesidade $^{19}$. No presente estudo a média do Índice de Massa Corporal da população avaliada mostrou sobrepeso, isso pode se justificar devido ao elevado consumo de alimentos industrializados, já que os mesmos são de fácil acesso por muitas vezes possuírem menor preço, no entanto esses produtos apresentam alto teor de gordura, açúcar simples e sódio ${ }^{20}$.

Ainda, olhando para o consumo alimentar, não foram encontradas associações com a EBIA. Acredita-se que esse resultado possa ser justificado em partes pelo baixo número de amostra, isso se dá mediante a pandemia, cenário atual vivenciado pela população, onde exige rígidas medidas de segurança, como por exemplo o uso de máscara, frequente higienização das mãos com álcool em gel, isolamento e distanciamento social a modo de evitar a contaminação causada pelo Coronavírus, fazendo com que os idosos apresentassem receio em receber visitas das pesquisadoras em seus domicílios ${ }^{19}$.

Outro estudo avaliou também a associação entre IA e consumo alimentar em idosos, demonstrando que a prevalência de IA nessa população é elevada, estando associada a diversos fatores principalmente ao consumo alimentar caracterizado pela ingestão inadequada de nutrientes ${ }^{22}$.

Observou-se que a média dos macronutrientes como o carboidrato e lipídio são semelhantes, independente do grau de IA. Foi encontrado no estudo de Mondini et. al a presença de idosos que apresentavam IG e possuíam uma ingestão calórica reduzida se comparado as demais classificações devido ao baixo consumo alimentar, porém, não foram encontrados estudos suficientes que relacionassem o teor calórico diário com o nível de IA.

A proteína foi o único macronutriente que apresentou pequenas mudanças de acordo com as classificações de IA, onde observa-se um consumo mais elevado nos indivíduos que apresentaram IL, seguido da IM e por fim IG. Um estudo que avaliou a IA e o consumo alimentar inadequado na cidade de São Leopoldo - RS, mostrou que os indivíduos classificados em IM ou IG possuem falta de recursos financeiros o que consequentemente leva a diminuição da capacidade de adquirir os alimentos, principalmente aqueles que são fontes de proteína, já que possuem um maior custo ${ }^{24}$.

As recomendações propostas pela Resource Description and Access - RDA para adultos e idosos é de 10 a $15 \%$ do valor calórico 
total de proteína, menos que $30 \%$ de lipídio e mais do que $50 \%$ de carboidratos ${ }^{25}$.

Esses dados podem parecer contraditórios, uma vez que se espera encontrar baixo peso em indivíduos que consomem baixas calorias. A presente pesquisa apresentou algumas limitações, como a quantidade reduzida do número de amostras, comprometimento na tomada das medidas antropométricas (peso, altura) e recordatório alimentar somente em 1 momento da pesquisa devido aos cuidados com a COVID-19 e exposição. Outro fator a ser destacado é a baixa participação de indivíduos do sexo masculino. Porém, esse estudo também apresenta pontos positivos, o tema estudado é de alta relevância e precisa ser considerado quando se fala em saúde pública.

De acordo com os desfechos apresentados, pode-se concluir que foi encontrado IA na maioria dos indivíduos avaliados, porém não houve associação entre IA e consumo alimentar de idosos assistidos por uma Estratégia da Saúde da Família (ESF) do interior paulista.

\section{CONFLITOS DE INTERESSE}

Os autores declaram não haver qualquer potencial conflito de interesse que possa interferir na imparcialidade deste trabalho científico.

\section{AGRADECIMENTOS}

Agradecemos a UNOESTE por fazer possível a execução desse trabalho e aos voluntários que aceitaram participar da pesquisa mesmo nesse momento tão delicado de pandemia.

\section{REFERÊNCIAS}

1. Pereira RJ. Nutrição e envelhecimento populacional: desafios e perspectivas. Rev Jour Heal NPEPS. 2019;4(1):1-5. DOI: http://dx.doi.org/10.30681/252610103714

2. IBGE. Censo Agro [Internet]. Rio de Janeiro, 2017 [acesso em 2021 jan 12] Disponível em:

https://agenciadenoticias.ibge.gov.br/agenciasala-de-imprensa/2013-agencia-

denoticias/releases/21837-projecao-daPopulacao-2018-numero-de-habitantes-dopaisdeve-parar-de-crescer-em-2047
3. Soares GFC, Andrade EGS. A osteoporose: um dos principais fatores responsável de fraturas em idosos e sua relevância. Rev Inic Cient Ext. 2019 [acesso em 2021 jan 12];2(1):1-6. Disponível em: https://revistasfacesa.senaaires.com.br/index.p $\mathrm{hp} /$ iniciacao-cientifica/article/view/138/92

4. Dias MF. Fatores que contribuem para adesão e desistência de um programa de atividades físicas para idosos. [Dissertação]. Brasília: Universidade de Brasília - Faculdade De Educação Física; 2018.

5. Volpini $M$, Frangella VS. Avaliação nutricional de idosos institucionalizados. Einstein (São Paulo).2013;11(1):32-40. DOI: https://doi.org/10.1590/S1679$\underline{45082013000100007}$

6. Souza JD, Martins MV, Franco FS, Martinho KO, Tinôco AL. Dietary patterns of the elderly: characteristics and association with socioeconomic aspects. Rev Bras de Geri e Geron. 2016;19(6):970-7. DOI: https://dx.doi.org/10.1590/1981$\underline{22562016019.160035}$

7. Kuwae CA, Carvalho MC da VS, Prado SD, Ferreira FR. Concepções de alimentação saudável entre idosos na Universidade Aberta da Terceira Idade da UERJ: normas nutricionais, normas do corpo e normas do cotidiano. Rev Bras de Geri e Gero. 2015;18(3):621-30. DOI: https://dx.doi.org/10.1590/1809-

$\underline{9823.2015 .14224}$

8. Filipa S. Insegurança alimentar e risco nutricional na pessoa idosa em centro de dia. [tese]. Aveiro: Universidade do Porto (Instituto de Ciências Biomédicas de Abel e da Universidade de Aveiro); 2018.

9. Ferreira E, Modesto NE, Santana SS. Segurança alimentar, perfil nutricional e de saúde de usuários de unidades básicas de Americana. Rev de Trab Acad da FAM. 2019; 4(1):7-15.

10. Trivellato PT, Morais D de C, Lopes SO, Miguel E da S, Franceschini S do CC, Priore SE. Insegurança alimentar e nutricional em famílias do meio rural brasileiro: revisão sistemática. 
Cien Saude Colet. 2019;24(3):865-74. DOI: https://dx.doi.org/10.1590/1413-

\subsection{7}

11. Paz AA, Santos LRB, Eidt RO. Vulnerabilidade e envelhecimento no contexto da saúde. Acta Paul Enferm.2006; 19(3): 338-42. DOI: $\quad$ http://dx.doi.org/10.1590/S0103$\underline{21002006000300014}$

12. Fechine BRA, Trompieri N. O processo de envelhecimento: as principais alterações que acontecem com o idoso com o passar dos anos. InterSciencePlace. 2012;1(20):10694. DOI: http://dx.doi.org/10.6020/1679-9844/2007

13. Paula NM, Zimmermann SA. A insegurança alimentar no contexto da pandemia da covid-19 no Brasil. Revista NECAT - Rev do Núc de Estu de Econ Cat. 2021;10(19):56-67.

14. Escala Brasileira de Insegurança Alimentar - EBIA: análise psicométrica de uma dimensão da Segurança Alimentar e Nutricional - Acervo Social. Estudo Técnico. 2014; 1(14):5-9.

15. Fisberg RM, Marchioni DM, Carolina A. Avaliação do consumo alimentar e da ingestão de nutrientes na prática clínica. Arq Bras Endocrinol Metab.2009;17(3):48-53.DOI: http://dx.doi.org/10.1590/S0004$\underline{27302009000500014}$

16. Ribeiro AC, Savio KEO, Rodrigues MLCF, Costa THM, Schmitz BAS. Validação de um questionário de frequência de consumo alimentar para população adulta. Rev Nutr Campinas. 2006;19(5):553-62.

17. Ferreira MG, Silva NF, Schmidt FD, Silva RMVG, Sichieri R, Guimarães LV, et al. Desenvolvimento de Questionário de Frequência Alimentar para adultos em amostra de base populacional de Cuiabá, Região CentroOeste do Brasil. Rev Bras Epidemiol.2010;13(3):413-24.

18. Fernandes SG. Insegurança alimentar em idosos a viver na comunidade em Portugal [Dissertação]. Lisboa: Universidade Nova de Lisboa - Escola Nacional de Saúde Pública; 2017.
19. Justo VC. Insegurança alimentar e fatores associados em idosos de Dourados [Dissertação]. Dourados: Universidade Federal da Grande Dourados; 2013.

20. Hammerschmidt KSA, Bonatelli LCS, Carvalho AA. Caminho da esperança nas relações envolvendo os idosos: olhar da complexidade sobre pandemia da covid-19. Rev Panam Salud Publica. 2020. DOI: https://doi.org/10.1590/1980-265X-TCE-2020$\underline{0132}$

21. Schlüssel MM, Silva AAM, Pérez ER, Kac G. Insegurança alimentar e excess de peso entre mulheres e crianças brasileiras. Cad Saúde Pública. 2012;29(2):219-226. DOI: https://dx.doi.org/10.1590/S0102$\underline{311 \times 2013000200003}$

22. Pedraza DF, Menezes TN de. Questionários de Frequência de Consumo Alimentar desenvolvidos e validados para população do Brasil: revisão da literatura. Cien Saude Colet. 2015;20(9):2697-720. DOI: https://dx.doi.org/10.1590/141381232015209.12602014

23. Ferreira MG, Silva NF da, Schmidt FD, Silva RMVG da, Sichieri R, Guimarães LV et al. Desenvolvimento de Questionário de Frequência Alimentar para adultos em amostra de base populacional de Cuiabá, Região CentroOeste do Brasil. Rev Bras de Epide. 2010;13(3):413-24. DOI: https://dx.doi.org/10.1590/141381232015209.12602014

24. Morais DC, Dutra LV, Franceschin SCC, Priore SE. Insegurança alimentar e indicadores antropométricos, dietéticos e sociais em estudos brasileiros: uma revisão sistemática. Ciênc Saúde Colet. 2014;19(5):1475-88. DOI: https://dx.doi.org/10.1590/141381232014195.13012013

25. Rosa TE da C, Mondini L, Gubert MB, Sato GS, Benício MHD. Segurança alimentar em domicílios chefiados por idosos, Brasil. Rev bras geriatr gerontol. (Online). 2012;15(1):69-77. DOI: $\quad$ https://doi.org/10.1590/1413$\underline{81232014195.13012013}$ 
26. Ruschel LF, Henn RL, Backes $V$, Melo $P$ de, Marques LA da S, Olinto MTA. Insegurança alimentar e consumo alimentar inadequado em escolares da rede municipal de São Leopoldo, RS, Brasil. Cien Saude Colet. 2016;21(7):227586. DOI: https://dx.doi.org/10.1590/1413$\underline{81232015217.00742015}$

25. Moreira APB, Alfenas GRC, Santana RFL, Priore ES, Franceschini CCS Evolução e interpretação das recomendações nutricionais para os macronutrientes. Rev Bras Nutr Clin. 2012;27(1): 51-9. 